\title{
CRISPR/Cas9 Knockout Strategies to Ablate CCAT1 IncRNA Gene in Cancer Cells
}

\author{
Khadijeh Zare ${ }^{1}$, Milad Shademan ${ }^{1}$, Mohammad M. Ghahramani Seno ${ }^{1,2}$ and Hesam Dehghani ${ }^{1,2,3^{*}}$ (D)
}

\begin{abstract}
Background: With the increasing discovery of long noncoding RNAs (IncRNAs), the application of functional techniques that could have very specific, efficient, and robust effects and readouts is necessary. Here, we have applied and analyzed three gene knockout (KO) strategies to ablate the CCAT1 gene in different colorectal adenocarcinoma cell lines. We refer to these strategies as "CRISPR excision", "CRISPR HDR", and "CRISPR du-HITI".

Results: In order to obstruct the transcription of IncRNA or to alter its structure, in these strategies either a significant segment of the gene is removed, or a transcription termination signal is inserted in the target gene. We use RT-qPCR, RNA-seq, MTT, and colony formation assay to confirm the functional effects of CCAT1 gene ablation in knockout colorectal adenocarcinoma cell lines. We applied three different CRISPR/Cas9 mediated knockout strategies to abolish the transcription of CCAT1 IncRNA. CCAT1 knockout cells displayed dysregulation of genes involved in several biological processes, and a significant reduction for anchorage-independent growth. The du-HITI strategy introduced in this study removes a gene segment and inserts a reporter and a transcription termination signal in each of the two target alleles. The preparation of donor vector for this strategy is much easier than that in "CRISPR HDR", and the selection of cells in this strategy is also much more practical than that in "CRISPR excision". In addition, use of this technique in the first attempt of transfection, generates single cell knockouts for both alleles.

Conclusions: The strategies applied and introduced in this study can be used for the generation of CCAT1 knockout cell lines and in principle can be applied to the deletion of other IncRNAs for the study of their function.
\end{abstract}

Keywords: CRISPR/Cas9, Knockout, Cancer cell, Long noncoding RNA, CCAT1

\section{Background}

Long non-coding RNAs (lncRNAs) play important roles in the regulation of transcription and post-transcriptional processes of coding and non-coding RNAs. Different mechanisms of function have been reported for lncRNAs including guiding chromatin modifiers to specific genomic loci, sequestering transcription factors, allosteric modulation of transcriptional regulatory proteins, alteration of nuclear domains, modulation of translation, modulation of mRNA stability, and working as competing endogenous RNAs [1-3]. Investigations on the role of lncRNAs in normal development and their aberrant functionality in different diseases underscore their importance in cellular

\footnotetext{
* Correspondence: dehghani@um.ac.ir

'Department of Basic Sciences, Faculty of Veterinary Medicine, Ferdowsi

University of Mashhad, Azadi Square, Mashhad, Iran

${ }^{2}$ Division of Biotechnology, Faculty of Veterinary Medicine, Ferdowsi

University of Mashhad, Azadi Square, Mashhad, Iran

Full list of author information is available at the end of the article
}

phenomena such as genomic imprinting, dosage compensation, pluripotency, and differentiation commitment [4]. These findings have proven lncRNAs to be important molecules with significant functions.

Functional experiments to investigate the roles of lncRNAs are highly needed. A plethora of experiments has been devised and implemented to investigate the function of IncRNAs. These experiments can be divided into three categories. The first category includes RNA interference (RNAi) and antisense oligonucleotides that aim at the destruction of transcribed lncRNA. The second category includes clustered regularly interspaced short palindromic repeats (CRISPR)- mediated interference (CRISPRi) and activation (CRISPRa) that target the regulatory regions of the gene for tuning the level of its transcription. And the third category includes programmable nucleases including zinc finger nucleases (ZFNs), transcription activator-like effector nucleases (TALENs), and CRISPR/Cas9 (CRISPR-associated protein-9 nuclease)

(c) The Author(s). 2018 Open Access This article is distributed under the terms of the Creative Commons Attribution 4.0 International License (http://creativecommons.org/licenses/by/4.0/), which permits unrestricted use, distribution, and 
that are used for the ablation of the gene. Different criteria have been described for proper selection of a functional experiment for a given transcript [5]. These criteria are mainly related to the location of the target lncRNA gene and its position relative to other genes, the location of its regulatory regions [5], and sub-cellular localization of lncRNA. Knockdown of a lncRNA as a strategy to study its function has at least two limitations: (1) the incomplete depletion of the transcript due to the nature of the approach and the nuclear localization of lncRNA, and (2) possible off target effects [6, 7]. When transcription of the target gene is regulated in a complicated fashion, application of CRISPRi and CRISPRa techniques may also fail to inhibit or induce the lncRNA transcription [7]. For many lncRNAs with complex transcriptional profile and regulatory regions, the CRISPR/Cas9 mediated ablation of the locus can act as a very specific and powerful tool to study their functions.

The colon cancer associated transcript 1 (CCAT1) lncRNA is upregulated in various human malignant and pre-malignant tissues [8] including colon adenocarcinoma [9], gastric carcinoma [10-12], ovarian cancer [13], hepatocellular carcinoma [14], and other kinds of cancers. CCAT1 is known to be involved in various normal and pathologic cellular processes such as proliferation, migration and metastasis [15-18]; it was shown to also act as a competing endogenous RNA [19, 20], and a regulator of cMYC $[15,21]$. However, due to the complexity of the processes that CCAT1 is known to control or contribute in, further investigations are required to clarify the exact molecular mechanism (s) by which this lncRNA acts. Gene knockdown procedures are helpful in delineating gene functions and RNAi [20-22] and antisense oligonucleotide [16] techniques have so far been used to study the CCAT1 functions. But, RNAi and antisense techniques act mostly within the cytoplasm, while many lncRNAs, including CCAT1, function mainly inside the nucleus and hence these techniques are far from being highly efficient [23].

In this work, we used three CRISPR/Cas9 mediated gene knockout strategies to deplete genomic CCAT1 transcription. The first strategy (herein named "CRISPR excision") involved the complete removal (excision) of a DNA segment encompassing an exon. In the second strategy, a DNA segment coding for a DNA reporter construct including transcription terminator, was inserted in an exonic region in CCAT1 genomic locus by homology-directed repair (HDR) mechanism (herein named "CRISPR HDR"). And in the final strategy, hereafter called "CRISPR du-HITI" standing for "CRISPR/ Cas9 dual allele homology-independent targeted integration", we employed a modified version of CRISPR/Cas9 HITI [24]. In this strategy, in each allele, the targeting fragment replaces the genomic region located between two double-strand breaks (DSBs) formed by Cas9. In addition, two alleles are targeted simultaneously by two different inserts, making it possible to select the targeted genomic locus by both alleles. We successfully applied and exploited these three knockout strategies and established model cell lines lacking CCAT1 lncRNA.

\section{Results \\ Application of Three Different CRISPR/Cas9 Mediated Knockout Strategies Targeting the CCAT1 IncRNA}

In the human $8 \mathrm{q} 24.21$ gene desert region, the CCAT1 gene $(\sim 11.8 \mathrm{~Kb})$ is located $\sim 173 \mathrm{~kb}$ downstream of the cancer susceptibility 21 (CASC21), and $\sim 31 \mathrm{~kb}$ upstream of the cancer susceptibility 19 (CASC19) gene loci. CCAT1 transcription is highly upregulated in the pre-malignant adenomatous polyps and malignant colorectal carcinoma [8]. Two short and long isoforms of CCAT1 have been identified and it seems that the short isoform might be derived from the long isoform [25]. The long isoform of CCAT1 is totally retained in the nucleus. However, all the experiments designed to explore CCAT1 functions have so far used either RNAi or antisense oligonucleotides. To develop colorectal adenocarcinoma cellular models that lack CCAT1 transcription, we used three different strategies to knockout CCAT1. Our aim here was to interfere with the normal function of CCAT1 by removing a segment of the gene important for the secondary structure of CCAT1, or by introducing a transcription termination signal within the CCAT1 locus to cause a premature transcription termination.

The first strategy, that we here call "CRISPR excision", involves precise deletion of a genomic fragment using two sgRNAs (Fig. 1a). In this strategy, we used two sgRNAs to direct the endonuclease activity of Cas9 to either side of CCAT1 exon 1 (Fig. 1a). For this purpose, we used HT-29, SW-480, and HCT-116 cell lines. After a first round of transfection and selection we obtained 45 HT-29 clones. PCR from genomic DNA revealed that 7 clones had one copy of CCAT1 deleted and no clones were homozygous for this deletion. We therefore used the heterozygous clones for a second round of "CRISPR excision" and after transfection and selection we were able to identify 2 out of 50 clones which were homozygous knockouts for CCAT1 as verified by PCR analysis of genomic DNA and sequencing of the PCR product (Additional file 1: Figure S1). RT-qPCR measurements of CCAT1 mRNA from the produced clones revealed a 370,000 fold (Fig. 2c) reduction of CCAT1 mRNA in the knockout clones compared to the wild-type cells. Previous reports achieved just a $\sim 10$ fold knockdown of CCAT1 in HT-29 cells using antisense oligonucleotides [25].

The second strategy that we here refer to as "CRISPR HDR" involved the insertion of a reporter gene and a 


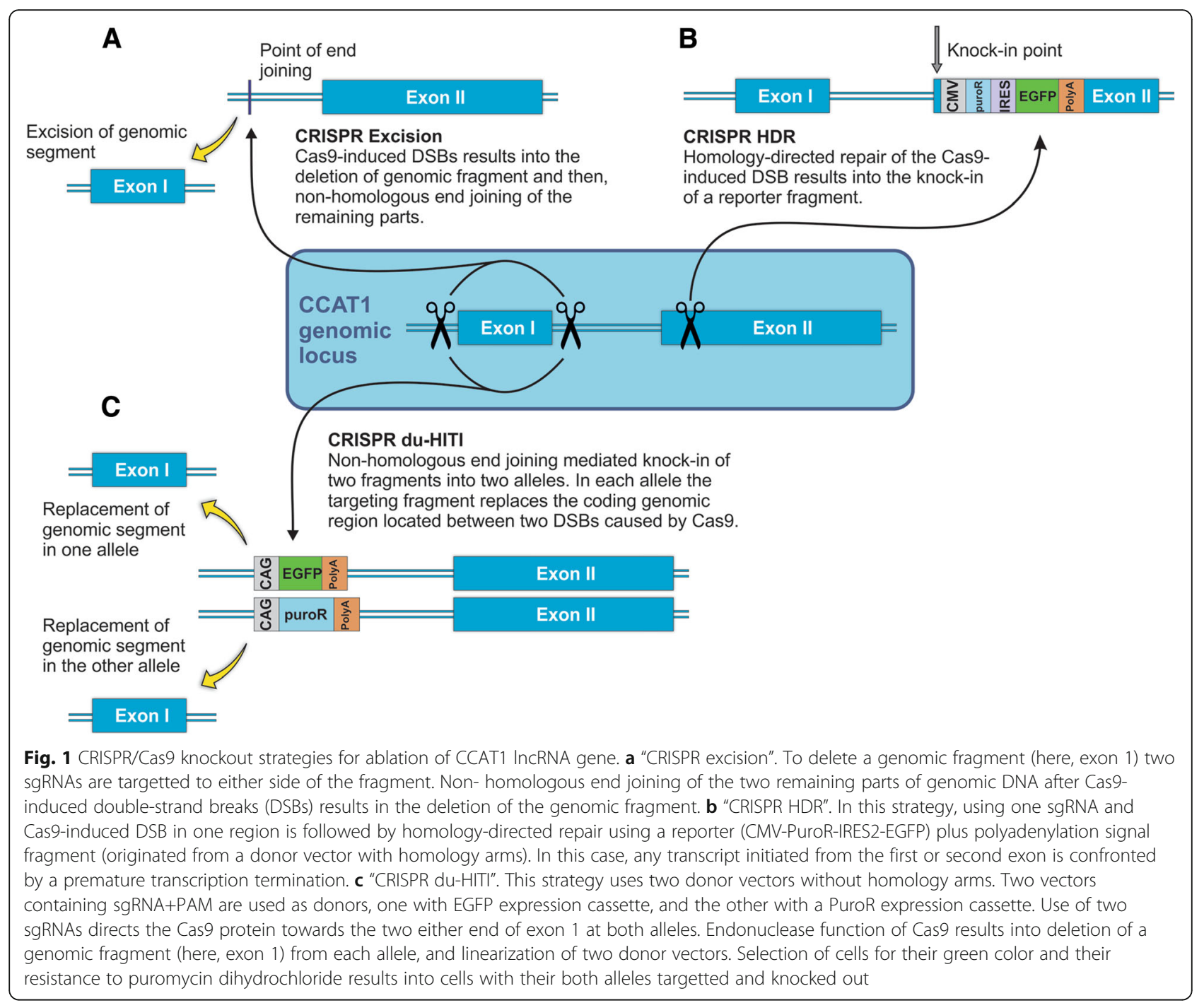

transcription termination signal in CCAT1 genomic locus using a single sgRNA and a donor vector with homology arms (Fig. 1b). We targeted the initial regions of the second exon since it has been reported that this region acts as a promoter for transcripts that originate from exon 2. The inclusion of a transcription termination signal in exon 2 ensures that the transcription of transcripts originating from exon 1 or from the proximal region of exon 2 will cease prematurely. Our reporter construct includes a green fluorescence protein (GFP) gene and DNA for the resistance to puromycin dihydrochloride to facilitate selection of correctly modified cells. In the first attempt of selection of puromycin resistant and green colonies, we selected $6 \mathrm{SW}-480$ and 14 HCT-116 clones. The selected clones were clonally expanded and were subjected to DNA extraction, PCR validation, and Sanger sequencing (Additional file 1: Figure S1). These analyses indicated that 4 out of 6 selected SW-480 and 8 out of 14 selected HCT-116 clones harbored the desired fragment insertions. RT-qPCR analysis of these clones revealed statistically significant lower expression levels ( 2 fold reduction) of CCAT1 compared to the control cells (Fig. 2d).

Our third strategy, here referred as "CRISPR du-HITI" comprises the deletion of a genomic fragment encompassing exon I of CCAT1 using two sgRNAs and insertion of two reporters and transcription termination signals using two donor vectors without homology arms (Fig. 1c). Cloning of the donor vectors used for this strategies was less laborious than that used for "CRISPR HDR" and the presence of a selection made the screening of correctly modified cells more practical compared to the "CRISPR excision" approach. In addition, using this strategy it is possible to mostly identify homozygous deletion of the target gene by virtue of selection for both GFP and puromycin. In the selected clones one allele will be therefore have the GFP reporter and the other allele the PuroR cassette. Furthermore, in this strategy, it is also possible to 
A

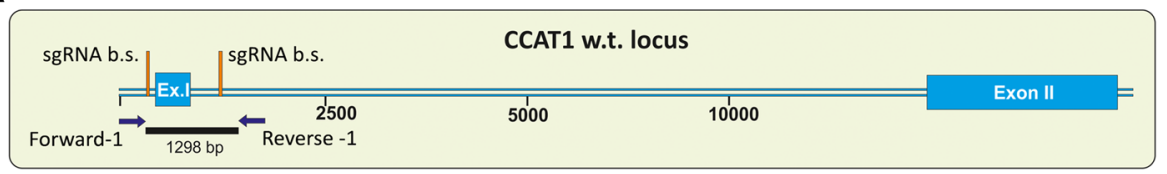

\begin{tabular}{|c|c|c|c|c|}
\hline \multirow{2}{*}{ sgRNA b.s. } & sgRNA b.s. & \multicolumn{2}{|c|}{ CRISPR Excision (exon I) } & \multirow{2}{*}{ Exon II } \\
\hline & $-\leftarrow \begin{array}{r}2500 \\
- \text { Reverse }-1\end{array}$ & 5000 & 10000 & \\
\hline
\end{tabular}

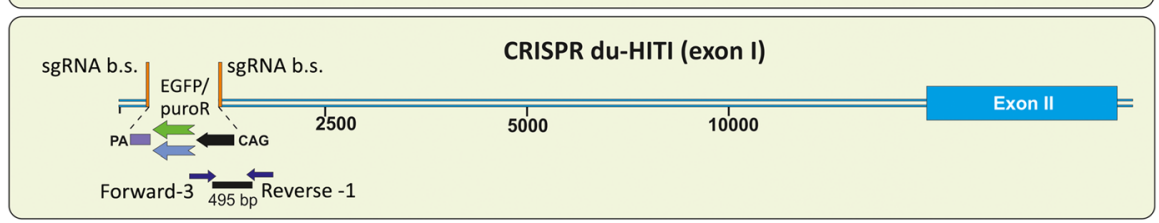

SgRNA b.s. CRISPR HDR (exon II)

B

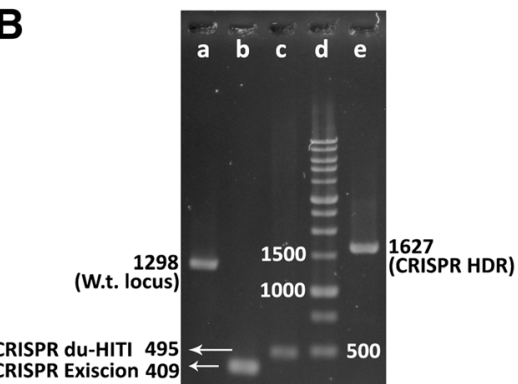

\section{D}

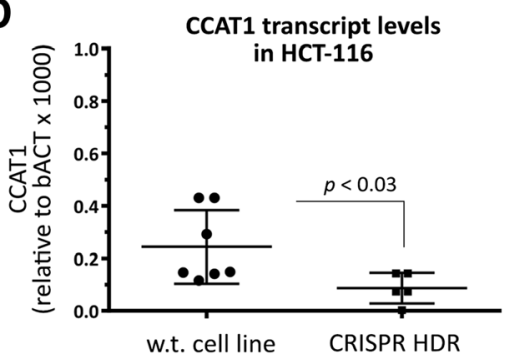

C

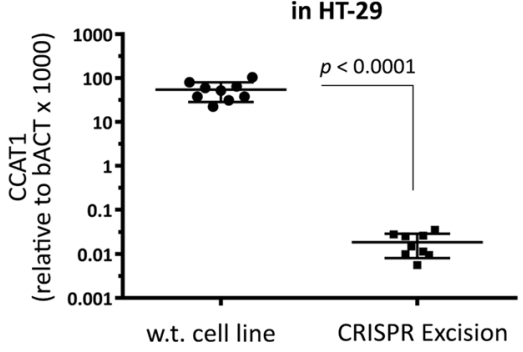

E

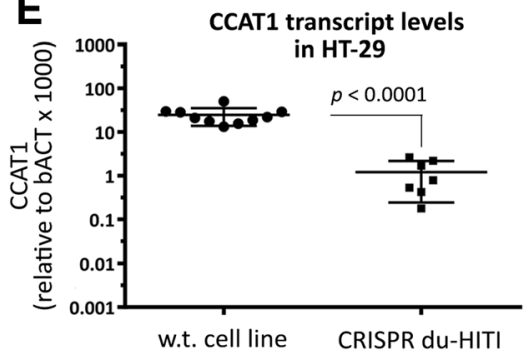

Fig. 2 Confirmation of the CCAT1 knockouts and qPCR analysis. a CCAT1 wild-type locus and its knockout alleles by "CRISPR excision", "CRISPR du-HITI", and "CRISPR HDR". Location of sgRNA binding sites (sgRNA b. s.), primers to perform PCR analysis (Table 4), and inserted fragments are shown. "CRISPR excision" of exon I (Ex. I) is confirmed by a 409 bp PCR fragment using two primers at either side of exon I. "CRISPR du-HITI" is confirmed by a PCR product of 495 bp using a primer in the insert and the other in the flanking region. The insert used for "CRISPR du-HITI" could express EGFP (bright green) or puroR (light blue) under CAG promoter. In this strategy when two alleles are targetted simultaneously, then the cells show both green fluorescence and resistance to puromycin dihydrochloride. "CRISPR HDR" was performed on exon Il using an insert containing DSred (red in color), CMV, puroR, IRES, GFP, PA (poly adenylation signal, purple in color). In "CRISPR HDR" strategy cells with only one targetted allele could show both green fluorescence and resistance to puromycin dihydrochloride. PCR analysis using a primer in the insert and the other in the flanking region results in a 1627 bp product (Table 4). b Gel electrophoresis of PCR fragments for wild-type (w.t.) (a), CRISPR excised (b; clone \# 20), "CRISPR du-HITI" knockout (c; clone \# 27), and "CRISPR HDR" alleles (e; clone \# 2i) according to the maps in panel A. Lane d is DNA size marker. c CCAT1 transcript relative to $\beta$-actin levels in wild-type and "CRISPR excision" knockout HT-29 colon adenocarcinoma cells. d CCAT1 transcript relative to $\beta$-actin levels in wild-type and "CRISPR HDR" knockout HCT-116 colon adenocarcinoma cells. e CCAT1 transcript relative to $\beta$-actin levels in wild-type and "CRISPR du-HITI" knockout SW-480 colon adenocarcinoma cells. In panels, C, D, and E the absolute copy number for CCAT1 and $\beta$-actin transcripts were quantified based on the related standard curves, and for three series of cell line CDNAs, the quantity of the CCAT1 transcript divided by the quantity of the $\beta$-actin was plotted. The statistical differences between the wild-type and knockout cell lines are analyzed by Mann-Whitney $U$ test 
delete large genomic fragments and replace them with different reporter cassettes. In the first attempt of transfection, single cell isolation, and clonal expansion, we were able to acquire 6 clones of HCT-116 cells that had permanent green fluorescence phenotype and puromycin resistance (Fig. 2). The knock-in was verified by PCR analysis that showed the insertion of the desired fragments (Additional file 1: Figure S1). These clones had statistically significant lower expression levels (27 fold reduction) of CCAT1 compared to the control cell line (Fig. 2e).

\section{CCAT1 Knockout Cells Display Dysregulation of Genes Involved in Several Biological Processes}

We used RNA-Seq to evaluate changes in the transcriptional landscape associated with inactivation of CCAT1 gene in colon adenocarcinoma cells (CCAT1 KO cells). We tested for enriched GO (Gene Ontology) terms in the wild-type versus knockout transcriptomes. Differentially expressed (DE) transcripts (log2-fold change $>1$ and $<-$ $1)$, statistically significant $(p<0.05)$ for enrichment in the CCAT1 $\mathrm{KO}$ cells, were identified. GO analysis indicated that a total of 332 genes were associated with some biological processes, molecular functions, or cellular components. The enriched GO terms in the category of molecular function, biological process, and cellular components are shown in Tables 1, 2 and 3.

\section{CCAT1 Knockout Cells Show Reduced Anchorage- Independent Growth}

The role of CCAT1 in anchorage-independent growth was analyzed by soft agar colony formation assay. It is known that inhibition of entosis enhances anchorage-independent growth in soft agar assay and promotes tumorigenesis [26].
HT-29, HCT-116, and SW-480 wild-type and CCAT1 KO cells were cultured in soft agar and allowed to form colonies over a period of 3 weeks. The wild-type HT-29 cells formed an average of $35 \pm 4$ colonies per microscopic fields, while CCAT1 KO HT-29 cells formed an average of $24 \pm 3$ colonies. The average colony size of wild-type SW-480 colonies was $20.86 \pm 0.9$, while the average size of CCAT1 KO SW-480 cells was $0.4 \pm 0.08$ pixel squared (Fig. 3a).

MTT assay was performed to investigate whether the decrease in the number and size of soft agar colonies is associated with changes in the metabolic activity of CCAT1 KO cells. MTT assay performed $2 \mathrm{~h}$ after cell seeding did not reveal any significant changes between $\mathrm{KO}$ and wild-type cells. However, MTT assay after $24 \mathrm{~h}$ exhibited a significant decrease in the absorbance readings in two CCAT1 $\mathrm{KO}$ cell lines compared to the wild-type cells (Fig. 3b). This suggested that the decreased MTT absorbance reading after $24 \mathrm{~h}$ might be caused by the lower number of cells in the knockout groups, while this effect is not evident when cells are cultured only for $2 \mathrm{~h}$ and have not started to divide [27, 28].

\section{Discussion}

With the increasing discovery of lncRNAs, there is a growing need for application of functional studies to reveal their biological roles. Similar to proteincoding genes, approaches aiming at expression inhibition are useful for studying lncRNA biological functions. However, the two approaches routinely exploited for this purpose, i.e. RNA interference and antisense strategies, are not as efficient for lncRNAs with nuclear localization. Furthermore, these techniques are associated with poor depletion of the target gene, off-target

Table 1 Enriched gene ontology terms in the CCAT1 wild-type versus CCAT1 knockout transcriptomes derived from differentially expressed genes (in the category of molecular function)

\begin{tabular}{|c|c|c|c|}
\hline$\overline{\mathrm{ID}}$ & Term & Gene Count & $p$ Value \\
\hline GO:0004867 & serine-type endopeptidase inhibitor activity & 9 & $2.50 \mathrm{E}-04$ \\
\hline GO:0016491 & oxidoreductase activity & 12 & $6.60 \mathrm{E}-04$ \\
\hline GO:0005515 & protein binding & 171 & $9.60 \mathrm{E}-03$ \\
\hline GO:0008236 & serine-type peptidase activity & 5 & $2.30 \mathrm{E}-02$ \\
\hline GO:0004879 & RNA polymerase II transcription factor activity, ligand-activated sequence-specific DNA binding & 4 & $2.30 \mathrm{E}-02$ \\
\hline GO:0004252 & serine-type endopeptidase activity & 10 & 3.10E-02 \\
\hline GO:0015485 & cholesterol binding & 4 & 3.30E-02 \\
\hline GO:0004029 & aldehyde dehydrogenase (NAD) activity & 3 & 3.30E-02 \\
\hline GO:0032810 & sterol response element binding & 2 & $3.40 \mathrm{E}-02$ \\
\hline GO:0003727 & single-stranded RNA binding & 4 & 3.70E-02 \\
\hline GO:0016620 & oxidoreductase activity, acting on the aldehyde or oxo group of donors, NAD or NADP as acceptor & 3 & 4.10E-02 \\
\hline GO:0004715 & non-membrane spanning protein tyrosine kinase activity & 4 & 4.40E-02 \\
\hline GO:0005506 & iron ion binding & 7 & 4.70E-02 \\
\hline GO:0003779 & actin binding & 10 & 4.90E-02 \\
\hline
\end{tabular}

${ }^{a}$ Only GO terms with $p$ value of less than 0.05 are shown 
Table 2 Enriched gene ontology terms in the CCAT1 wild-type versus CCAT1 knockout transcriptomes derived from differentially expressed genes (in the category of biological process)

\begin{tabular}{|c|c|c|c|}
\hline $\mathrm{ID}$ & Term & Gene Count & $p$ Value $^{a}$ \\
\hline GO:0042493 & response to drug & 17 & $4.50 \mathrm{E}-05$ \\
\hline GO:0055114 & oxidation-reduction process & 24 & 1.30E-04 \\
\hline GO:0031295 & $\mathrm{T}$ cell costimulation & 7 & 1.80E-03 \\
\hline GO:0008283 & cell proliferation & 15 & $3.00 \mathrm{E}-03$ \\
\hline GO:0030522 & intracellular receptor signaling pathway & 5 & 3.40E-03 \\
\hline GO:0010951 & negative regulation of endopeptidase activity & 8 & 3.90E-03 \\
\hline GO:0090090 & negative regulation of canonical Wnt signaling pathway & 9 & 5.60E-03 \\
\hline GO:0008285 & negative regulation of cell proliferation & 15 & $6.00 \mathrm{E}-03$ \\
\hline GO:0006805 & xenobiotic metabolic process & 6 & 9.20E-03 \\
\hline GO:0051897 & positive regulation of protein kinase $B$ signaling & 6 & 1.20E-02 \\
\hline GO:0030449 & regulation of complement activation & 4 & 1.30E-02 \\
\hline GO:0006081 & cellular aldehyde metabolic process & 3 & $1.30 \mathrm{E}-02$ \\
\hline GO:0048013 & ephrin receptor signaling pathway & 6 & 1.40E-02 \\
\hline GO:0001822 & kidney development & 6 & 1.40E-02 \\
\hline GO:0007612 & learning & 5 & $1.40 \mathrm{E}-02$ \\
\hline GO:0032091 & negative regulation of protein binding & 5 & 1.40E-02 \\
\hline GO:0046685 & response to arsenic-containing substance & 3 & 1.90E-02 \\
\hline GO:0007568 & aging & 8 & $1.90 \mathrm{E}-02$ \\
\hline GO:0060337 & type I interferon signaling pathway & 5 & 2.10E-02 \\
\hline GO:0042632 & cholesterol homeostasis & 5 & 2.10E-02 \\
\hline GO:0043524 & negative regulation of neuron apoptotic process & 7 & $2.20 \mathrm{E}-02$ \\
\hline GO:0006954 & inflammatory response & 13 & 2.30E-02 \\
\hline GO:0016477 & cell migration & 8 & $2.40 \mathrm{E}-02$ \\
\hline GO:0006636 & unsaturated fatty acid biosynthetic process & 3 & $2.50 \mathrm{E}-02$ \\
\hline GO:0032570 & response to progesterone & 4 & 2.60E-02 \\
\hline GO:0038083 & peptidyl-tyrosine autophosphorylation & 4 & 2.80E-02 \\
\hline GO:0008360 & regulation of cell shape & 7 & 2.80E-02 \\
\hline GO:0032526 & response to retinoic acid & 4 & $3.00 \mathrm{E}-02$ \\
\hline GO:0002223 & stimulatory C-type lectin receptor signaling pathway & 6 & $3.00 \mathrm{E}-02$ \\
\hline GO:0003057 & regulation of the force of heart contraction by chemical signal & 2 & $3.30 \mathrm{E}-02$ \\
\hline GO:0045785 & positive regulation of cell adhesion & 4 & $3.30 \mathrm{E}-02$ \\
\hline GO:0042127 & regulation of cell proliferation & 8 & $3.40 \mathrm{E}-02$ \\
\hline GO:0031668 & cellular response to extracellular stimulus & 3 & $3.50 \mathrm{E}-02$ \\
\hline GO:0009615 & response to virus & 6 & $3.50 \mathrm{E}-02$ \\
\hline GO:0030325 & adrenal gland development & 3 & $3.80 \mathrm{E}-02$ \\
\hline GO:0071222 & cellular response to lipopolysaccharide & 6 & $3.90 \mathrm{E}-02$ \\
\hline GO:0030154 & cell differentiation & 14 & 4.30E-02 \\
\hline GO:0014070 & response to organic cyclic compound & 4 & 4.70E-02 \\
\hline GO:0070668 & positive regulation of mast cell proliferation & 2 & 4.90E-02 \\
\hline GO:0060675 & ureteric bud morphogenesis & 2 & 4.90E-02 \\
\hline GO:0006991 & response to sterol depletion & 2 & 4.90E-02 \\
\hline
\end{tabular}


Table 3 Enriched gene ontology terms in the CCAT1 wild-type versus CCAT1 knockout transcriptomes derived from differentially expressed genes (in the category of cellular component)

\begin{tabular}{llll}
\hline ID & Term & Gene Count & $p$ Value \\
\hline GO:0070062 & extracellular exosome & 78 & $4.50 \mathrm{E}-06$ \\
GO:0005615 & extracellular space & 42 & $1.30 \mathrm{E}-04$ \\
GO:0005829 & cytosol & 78 & $1.10 \mathrm{E}-03$ \\
GO:0005576 & extracellular region & 42 & $4.20 \mathrm{E}-03$ \\
GO:0031234 & extrinsic component of cytoplasmic side of plasma membrane & 6 & $5.50 \mathrm{E}-03$ \\
GO:0002102 & podosome & 4 & $7.20 \mathrm{E}-03$ \\
GO:0005856 & cytoskeleton & 14 & $9.80 \mathrm{E}-03$ \\
GO:0030687 & preribosome, large subunit precursor & 4 & $1.20 \mathrm{E}-02$ \\
GO:0031528 & microvillus membrane & 3 & $3.90 \mathrm{E}-02$ \\
GO:0005886 & plasma membrane & 83 & $4.10 \mathrm{E}-02$ \\
GO:0072562 & blood microparticle & 7 & $4.20 \mathrm{E}-02$ \\
GO:0034666 & integrin alpha2-beta1 complex & 2 & $4.90 \mathrm{E}-02$ \\
\hline
\end{tabular}

${ }^{\mathrm{a} O n l y ~ G O}$ terms with $p$ value of less than 0.05 are shown

effects, and technical variations. Applying newly developed genome editing approaches to alter lncRNA genomic locus so that the related IncRNA becomes non-functional is a good alternative. However, considering the non-coding nature of lncRNAs, changes at the corresponding genomic loci need to be large enough to adversely affect the structure and biological function of the expressed RNA. For example, a simple excision followed by NHEJ may not provide enough structural changes in a given lncRNA. Here, we used three different CRISPR-mediated KO strategies, i.e. "CRISPR excision", "CRISPR HDR", and "CRISPR du-HITI", to alter the CCAT1 locus in different colorectal adenocarcinoma cell lines.

"CRISPR excision" using two sgRNAs to delete a genomic fragment of lncRNA genes has already been reported [29-31]. For the implementation of this strategy, no donor vectors are used and thus, no reporter genes are inserted into the genome. As a result, the selection of KO cells becomes a tedious job. "CRISPR HDR", in contrast, uses donor vectors and thus, enables the user to select the targeted cells based on fluorescence and/or resistance to an antibiotic [30, 31]. However, in this strategy, homology arms need to be cloned into the donor vector. The "CRISPR du-HITI" which doesn't rely on the presence of homology arms, makes the selection of the target cells easier (Fig. 1). This approach enables us to direct fabrication of KOs so that each cell with both antibiotic resistance and fluorescence phenotypes is easily selected as a dual allele KO.

CCAT1 $\mathrm{KO}$ cells created in this study were verified by genomic PCR, sequencing, and RT-qPCR (Fig. 2). Since the qPCR forward and reverse primers were complementary to exon 1 and exon 2, respectively, we did not expect to have any amplification products from "CRISPR excision" and "CRISPR du-HITI" KO cells. In these two approaches, exon 1 was completely removed. However, in "CRISPR HDR" the first segment of exon 2 (after the complementary sequence to reverse primer) was targeted with a fragment containing reporter/transcription termination signal. Thus, an amplification product was expected. This could explain the presence of RNA which was still measurable in these cells, and that only the two-fold CCAT1 decrease was observed (Fig. 2d). These three cell lines express different levels of CCAT1, the highest level of CCAT1 is detected in HT-29, while both SW-480 and HCT 116 express low levels of CCAT1 (Additional file 2: Figure S2). Presence of a residual RNA (the cycle threshold for knockout CCAT1 in all cell lines) could also be attributed to the cancerous nature of these cells and the presence of CCAT1 extra copies and pseudogenes in the genome.

The CCAT1 KO cells were subjected to RNA-seq, soft agar colony formation assay, and MTT analysis. These cells displayed differential expression of a number of genes (Tables 1-3). Accordingly, soft agar colony formation and MTT assays were able to verify that cell proliferation is negatively affected in the CCAT1 KO cells (Fig. 3). This finding in turn, is in accordance with the previous reports that CCAT1 suppression affects genes that are involved in the regulation of cell proliferation [32].

\section{Conclusions}

In conclusion, we characterized here three CRISPR/Cas9 mediated strategies that provide effective tools for IncRNA functional surveys. Furthermore, the CCAT1 KO cell lines created in this study can be used for further functional analyses to reveal the battery of functions of CCAT1. The du-HITI strategy introduced in this study is easy to implement and can be applied for the generation of homozygous cells knockout for a specific lncRNA of interest. 

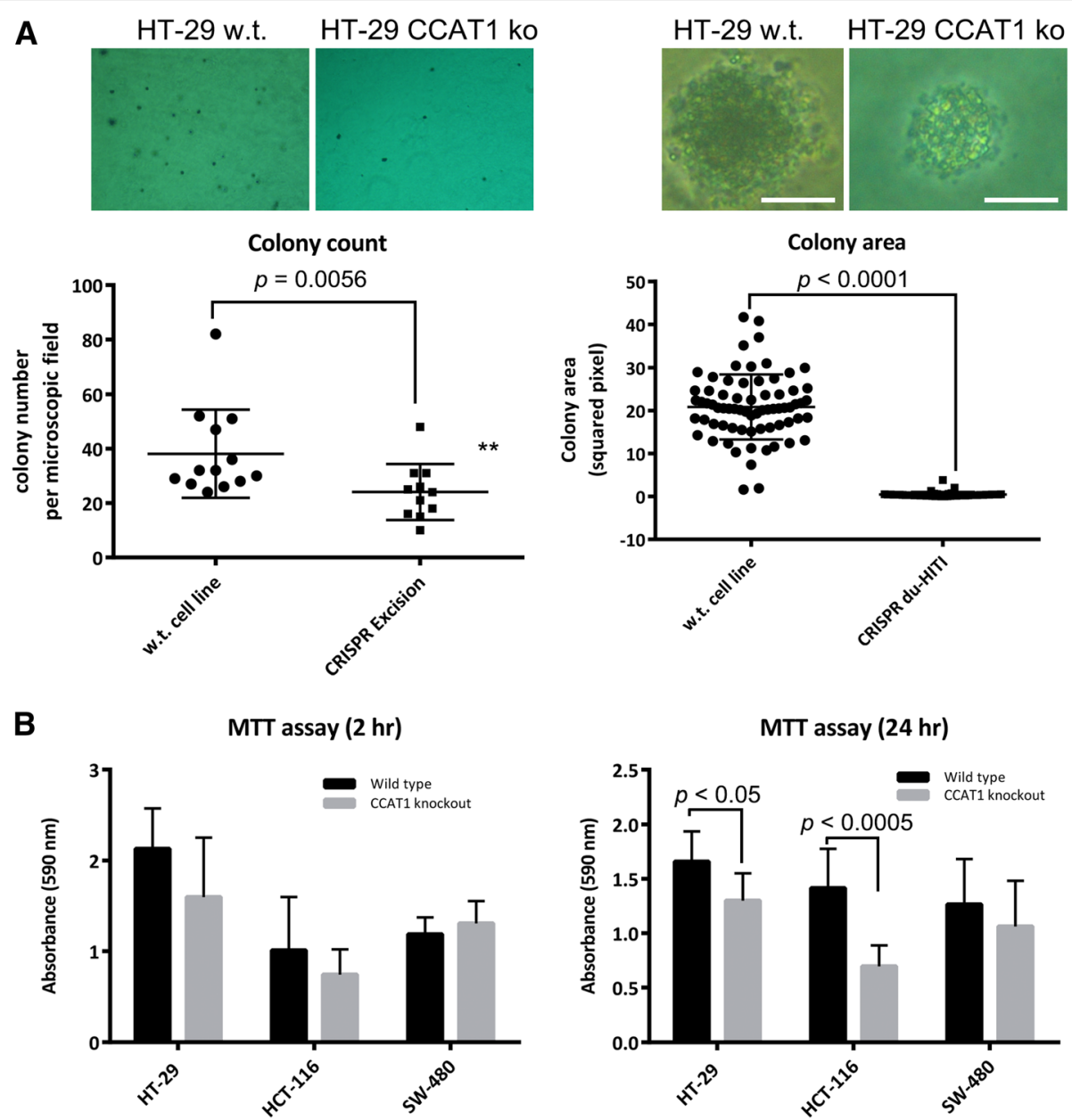

Fig. 3 CCAT1 knockout cells lose the capacity for anchorage-independent growth. a Representative images of soft agar colony formation assay for wild-type and CCAT1 KO cell lines and evaluation of colony count and colony area in these lines. b MTT assay in HT-29, HCT-116, and SW-480 wild-type and CCAT1 KO cell lines performed $2 \mathrm{~h}$ and $24 \mathrm{~h}$ after seeding. The statistical differences between the wild-type and knockout cell lines are analyzed by Mann-Whitney test. The scale bars in panel A denote $200 \mu \mathrm{m}$

\section{Methods}

\section{Cell Culture}

All cell lines were purchased from the National Cell Bank of Iran (Pasteur Institute, Iran) and cultured as recommended by the ATCC. Three colorectal adenocarcinoma cells were used, which differed based on microsatellite stability (MS) and CpG island methylator phenotype (CIMP). HT-29 cells are microsatellite stable (MSS), and CIMP high, SW-480 cells are MSS and CIMP low, and HCT-116 cells show microsatellite instability (MSI) and are CIMP high [33]. HT-29 and SW-480 cells were cultured in RPMI (Biowest, France) supplemented with 10\% FBS (Gibco, USA), $50 \mathrm{U} / \mathrm{ml}$ penicillin and $50 \mu \mathrm{g} / \mathrm{ml}$ streptomycin (Sigma-Aldrich, USA) at $37^{\circ} \mathrm{C}$ in $5 \% \mathrm{CO} 2$. HCT-116 cells were cultured in DMEM (Gibco, USA) containing $10 \%$ FBS and penicillin-streptomycin $(50 / 50 \mathrm{U} / \mathrm{ug} / \mathrm{ml})$ at $37{ }^{\circ} \mathrm{C}$ in a $5 \% \mathrm{CO} 2$ environment.

\section{DNA Constructs and Gene Targeting}

The single guide RNA (sgRNA) sequences targeting different segments of CCAT1 gene were designed using the CRISPR design tool (http://crispr.mit.edu/). Three vectors of pX459 (containing U6 promoter-sgRNA insertion site-sgRNA scaffold, and CAG promoter-Cas9-T2Apuromycin $\mathrm{N}$-acetyltransferase gene-bovine growth hormone polyadenylation signal), pX460-1 (containing U6 promoter-sgRNA insertion site-sgRNA scaffold, and CAG promoter-enhanced GFP (EGFP)-bovine growth hormone polyadenylation signal), and pX461-1 (containing U6 promoter-sgRNA insertion site-sgRNA scaffold, and CAG promoter-puromycin N-acetyltransferase (PuroR)-bovine growth hormone polyadenylation signal) were used for sub-cloning of sgRNAs. For this purpose, oligonucleotides (Table 4) containing the sgRNA expressing sequence and BbsI steaky ends were synthesized 
Table 4 Oligonucleotides used in this study

\begin{tabular}{|c|c|c|c|}
\hline Gene & Sequence (5' to $\left.3^{\prime}\right)$ & Product (bP) & Application \\
\hline \multirow[t]{11}{*}{ CCAT1 (NR_108049.1) } & $\begin{array}{l}\text { F: CTGACAACATCGACTTTG } \\
\text { R: CTCACAGTITCAAGGGA } \\
\text { Probe: FAM-CTTAGCCATACAGAGCCAACCTG-BHQ1 }\end{array}$ & 108 & qPCR \\
\hline & $\begin{array}{l}\text { F: CGATCGttctgttttcaatggggatt } \\
\text { R: TCGAGggagctgcggataacagcatat }\end{array}$ & 546 & Cloning of left homology arm \\
\hline & $\begin{array}{l}\text { F: CTAGTCCCgcatcacagctactgtcaaccc } \\
\text { R: ATCCCCtcaaagcacttctgtggtagga }\end{array}$ & 832 & Cloning of right homology arm \\
\hline & $\begin{array}{l}\text { Forward-1: CACATGGCTCCCATCACACTA } \\
\text { Reverse-1: GGGGGAAGAAATTTAAGATGCACA }\end{array}$ & 1298 (w.t.) 409 (ko) & $\begin{array}{l}\text { PCR confirmation of CRISPR Excision } \\
\text { knockout allele }\end{array}$ \\
\hline & $\begin{array}{l}\text { Forward-2: CACGCAGATCACATGACCCT } \\
\text { Reverse-2: CGGGCCATTACCGTAAG }\end{array}$ & 1627 (ko) & PCR confirmation of CRISPR HDR knockout allele \\
\hline & $\begin{array}{l}\text { Forward-2: CACGCAGATCACATGACCCT } \\
\text { R: AAACgatggagctgcggataacagC }\end{array}$ & 843 (w.t.) & PCR confirmation of CRISPR HDR wild-type allele \\
\hline & $\begin{array}{l}\text { Forward-3: CGGGCCATTTACCGTAAG } \\
\text { Reverse-1: GGGGGAAGAAATTTAAGATGCACA }\end{array}$ & 495 (ko) & $\begin{array}{l}\text { PCR confirmation of CRISPR du-HITI } \\
\text { knockout allele }\end{array}$ \\
\hline & $\begin{array}{l}\text { F: CACCGaatcggagtccaaagccatt } \\
\text { R: AAACaatggctttggactccgattC }\end{array}$ & - & sgRNA (downstream of Exon 1) \\
\hline & $\begin{array}{l}\text { F: CACCGataatggaggggatttacgt } \\
\text { R: AAACacgtaaatcccctccattatC }\end{array}$ & - & sgRNA (upstream of Exon 1) \\
\hline & $\begin{array}{l}\text { F: CACCGctgttatccgcagctccatc } \\
\text { R: AAACgatggagctgcggataacagC }\end{array}$ & - & sgRNA (Exon 2) \\
\hline & $\begin{array}{l}\text { F: CACCGaatggctttggactccgatttgg } \\
\text { R: AAACccaaatcggagtccaaagccattC }\end{array}$ & - & sgRNA bait (+PAM) (downstream of Exon 1) \\
\hline b-Actin & $\begin{array}{l}\text { F: TGCAGAAGGAGATCACTG } \\
\text { R: CTTGCTGATCCACATCTG } \\
\text { Probe: CY5-AAGATCAAGATCATTGCTCCTCCTGA-BHQ2 }\end{array}$ & 141 & $\mathrm{qPCR}$ \\
\hline
\end{tabular}

(Macrogen Inc., South Korea), annealed, phosphorylated and ligated into the BbsI-digested and gel purified (using Gel Extraction Kit; DENAzist Asia Co., Iran) vectors. For "CRISPR du-HITI" targeting vectors, the PAM sequence was also introduced after the sgRNA expressing sequence.

The vector used for "CRISPR HDR" targeting contained left homology arm (546 bp), DsRed2, herpes simplex virus thymidine kinase polyadenylation signal, CMV promoter, PuroR, IRES2, EGFP, SV40 polyadenylation signal, and right homology arm (832 bp).

According to Table 5, constructs (verified by Sanger sequencing; Macrogen Inc., South Korea) were used for transfection of colorectal adenocarcinoma cell lines using Lipofectamine 2000 reagent (Thermo Fisher Scientific, USA), Exgen 500 (Thermo Fisher Scientific, USA) or Polyethylenimine (Sigma-Aldrich, USA). For "CRISPR excision" and removal of CCAT1 exon 1, 2 weeks after transfection cells were cultured in low density, and individual colonies (50 colonies) were allowed to expand and then selected by PCR analysis. Selection of colonies for "CRISPR du-HITI" and "CRISPR HDR" was performed by their GFP expression and resistance to puromycin dihydrochloride (Sigma-Aldrich, USA). All "CRISPR excision", "CRISPR du-HITI", and "CRISPR HDR" individual colonies were verified by genomic DNA isolation, PCR analysis, and Sanger sequencing (Macrogen Inc., South Korea).

\section{Genomic DNA Isolation and Analysis}

Genomic DNA was isolated with the Genomic DNA Isolation Kit I (DENAzist Asia Co., Iran) from wild-type and knockout cell lines and was subjected to PCR amplification (Fig. 2). PCR-amplified bands after clean-up and reaction recovery (DENAzist Asia Co., Iran), were subjected to Sanger sequencing (Macrogen Inc., South Korea).

\section{Reverse Transcription Quantitative PCR}

Total RNA was isolated from wild-type and knockout cell lines using Total RNA Isolation Kit (DENAzist Asia Co., Iran). The quality and quantity of extracted RNA were evaluated using gel electrophoresis and a 2000 Nanodrop spectrophotometer (Thermo Scientific, USA). Total RNA $(1 \mu \mathrm{g})$ was reverse transcribed using random hexamer primers and MMLV reverse transcriptase (Thermo Fisher Scientific, USA). To quantify the level of transcripts for CCAT1 and $\beta$-actin, quantitative RT-PCR reactions containing Premix Ex Taq (Probe qPCR) master mix (Takara, Japan), $2 \mu \mathrm{l}$ cDNA template and each primer at $500 \mathrm{nM}$ and $100 \mathrm{nM}$ probe (dual-labeled hybridization probes, 5'FAM-3'BHQ1-labeled for CCAT1 and 5'CY5-3'BHQ2 for $\beta$-actin) in a $20 \mu \mathrm{l}$ reaction volume, were carried out in a Rotor-Gene Q real-time PCR cycler (Qiagen, USA). Amplification steps were: $95{ }^{\circ} \mathrm{C}$ for $5 \mathrm{~min}$, followed by 40 cycles of $94{ }^{\circ} \mathrm{C}$ for $30 \mathrm{~s}, 57.5^{\circ} \mathrm{C}$ for $30 \mathrm{~s}$, and $72{ }^{\circ} \mathrm{C}$ for 
Table 5 DNA constructs used in this study

\begin{tabular}{lll}
\hline Construct & Features & Application \\
\hline pX459_2 & hU6 promoter- sgRNA (downstream of CCAT1 Exon & CRISPR Excision \\
& 1)-sgRNA scaffold-CAG promoter-Cas9-T2A- PuroR-bGH polyA & CRISPR du-HITI \\
pX459_3 & hU6 promoter- sgRNA (upstream of CCAT1 Exon & CRISPR Excision \\
& 1)-sgRNA scaffold-CAG promoter-Cas9-T2A- PuroR-bGH polyA & CRISPR du-HITI \\
pX459_13 & hU6 promoter- sgRNA (CCAT1 Exon 2)-sgRNA scaffold-CAG promoter-Cas9-T2A- PuroR-bGH polyA & CRISPR HDR \\
pHD_4317_CCAT1 E2 HAs & Exon 2 LHA-DsRed2-HSV TK polyA-CMV promoter -PuroR-IRES2-EGFP-SV40 polyA-Exon 2 RHA & CRISPR HDR \\
pX460_11 & hU6 promoter-sgRNA (downstream of CCAT1 Exon 1) plus PAM-sgRNA scaffold-CAG & CRISPR du-HITI \\
& promoter-EGFP-bGH polyA & \\
pX461_11 & hU6 promoter-sgRNA (downstream of CCAT1 Exon 1) plus PAM-sgRNA scaffold-CAG & \\
& promoter-PuroR-bGH polyA & CRISPR du-HITI \\
& & \\
\end{tabular}

hU6 human U6 promoter, sgRNA single guide RNA, PuroR puromycin N-acetyltransferase, bGH bovine growth hormone polyadenylation signal, SV40 polyA SV40 polyadenylation signal, IRES internal ribosome entry site

$30 \mathrm{~s}$. To confirm the identity of PCR products, Sanger sequencing was performed (Macrogen Inc., South Korea).

Amplified fragments were sub-cloned in pTZ57R or pGATA plasmids and their serial dilution was used to make standard curves. Each dilution was subjected to three PCR reactions and real-time readings were performed in triplicate. Then, the log of copy numbers was plotted against cycle threshold $(\mathrm{Ct})$ numbers. For each qPCR reaction, efficiency $(E)$ was calculated from the calculated slope of standard curves, generated using 5 fold serially diluted solutions of plasmids, according to the following equation: $\mathrm{E}=\left(10^{-1 / \mathrm{slope}^{-1}}\right) \times 100 \%$. All standard curves were linear in the analyzed range with an acceptable correlation coefficient $\left(R^{2}\right)$. Absolute copy number for CCAT1 and $\beta$-actin transcripts were quantified based on the related standard curves. For three series of cDNAs, the quantity of the target transcript (CCAT1) was divided by the quantity of the reference gene $(\beta$-actin) and plotted.

\section{RNA Sequencing and GO Analysis}

Tuxedo pipeline [34] was performed for differential expression analysis of whole transcriptome sequencing results of HT-29 wild-type and knockout cell lines. The differentially expressed genes from the study were input for assessing the enrichment. For data precision and consistency, we adjusted $p$-value to correct the data, fold change was declared at least \pm 1 fold and $p$-value $<0.05$ was regarded as significant. Compared to the wild-type cell lines, 182 differentially expressed genes were selected and association of gene list to Gene Ontology (GO) terms was performed using the new GO category (GO Direct) of the latest released version of DAVID web tool (DAVID 6.8 Oct. 2016), (http://david.ncifcrf.gov) [35, 36].

\section{Soft Agar Colony Formation and MTT Assays}

The methodology previously described was followed [37]. To evaluate the anchorage-independent growth of
CCAT1 KO cell lines, cells were plated at $2 \times 10^{4}$ in DMEM/F12 containing 5\% horse serum, penicillin/ streptomycin, and 0.3\% 2-hydroxyethyl agarose (Sigma, USA) (with $0.6 \%$ 2-hydroxyethyl agarose underlay) in flat-bottom non-treated six-well plates (SPL, South Korea). By overlaying $1 \mathrm{ml}$ of $0.3 \%$ 2-hydroxyethyl agarose/medium solution onto the existing feeder layer, the feeding procedure was performed for three times (once a week). After 3 weeks, the colonies were fixed with $4 \%$ paraformaldehyde (Electron Microscopy Sciences, USA) in PBS for 30 min and stained with $0.5 \%$ methylene blue in ethanol for $30 \mathrm{~min}$. The number and area of colonies were determined using NIH ImageJ software [38].

To perform MTT (3-(4,5-Dimethylthiazol-2-Yl)-2,5Diphenyltetrazolium Bromide) assay, Cells were seeded in a 96-well plate in three or more replicates (5000 cells/ well). and MTT (to a final concentration of $0.5 \mathrm{mg} / \mathrm{ml}$ in culture medium) was added to each well after 2 or $24 \mathrm{~h}$ of seeding. The plate was incubated at $37^{\circ} \mathrm{C}$ in dark for $3 \mathrm{~h}$. Then, the media was discarded, $100 \mu \mathrm{l}$ of DMSO was added to each well, incubated at room temperature in the dark for $15 \mathrm{~min}$, and the color developed was measured at $590 \mathrm{~nm}$ using Epoch 2 microplate reader (BioTek Instruments Inc., USA).

\section{Additional files}

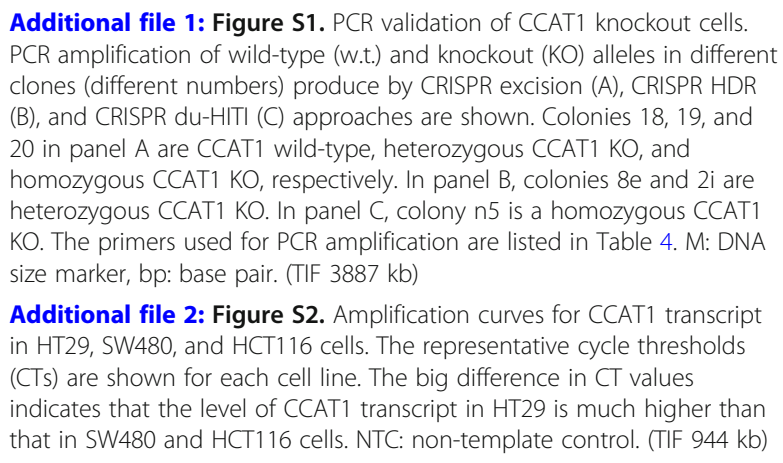

Additional file 1: Figure S1. PCR validation of CCAT1 knockout cells. PCR amplification of wild-type (w.t.) and knockout (KO) alleles in different clones (different numbers) produce by CRISPR excision (A), CRISPR HDR (B), and CRISPR du-HITI (C) approaches are shown. Colonies 18, 19, and 20 in panel A are CCAT1 wild-type, heterozygous CCAT1 KO, and homozygous CCAT1 KO, respectively. In panel B, colonies $8 \mathrm{e}$ and $2 \mathrm{i}$ are heterozygous CCAT1 KO. In panel C, colony n5 is a homozygous CCAT1 KO. The primers used for PCR amplification are listed in Table 4. M: DNA size marker, bp: base pair. (TIF 3887 kb)

Additional file 2: Figure S2. Amplification curves for CCAT1 transcript in HT29, SW480, and HCT116 cells. The representative cycle thresholds (CTs) are shown for each cell line. The big difference in $C T$ values indicates that the level of CCAT1 transcript in HT29 is much higher than that in SW480 and HCT116 cells. NTC: non-template control. (TIF 944 kb) 


\section{Abbreviations}

CCAT1: Colon cancer associated transcript 1; IncRNA: Long noncoding RNA: KO: Knockout; CRISPR: Clustered regularly interspaced short palindromic repeats; Cas9: CRISPR-associated protein-9 nuclease; HDR: Homology-directed repair; HITI: Homology-independent targeted integration; du-HITI: Dual allele HITI; RNAi: RNA interference; DSBs: Double-strand breaks; DE: Differentially expressed; RT: Reverse transcription; qPCR: Quantitative PCR

\section{Acknowledgements}

We dedicate this work to our deceased colleague, Dr. Mohammad Reza Bassami, for his help to conceptualize the early stages of this study.

\section{Funding}

This study was financially supported by grant number 93034176 from Iran National Science Foundation, grant number 942331 from National Institute for Medical Research Development of Iran, and grant number 33100 from Ferdowsi University of Mashhad, Iran.

\section{Availability of Data and Materials}

The datasets used and/or analysed during the current study are available from the corresponding author on reasonable request.

\section{Authors' Contributions}

$H D, K Z$, and MMGS conceived and designed the experiments. KZ and MS performed the experiments. KZ, MS, and HD analyzed the data and wrote the manuscript. HD supervised the experiments. All authors read and approved the final manuscript.

\section{Ethics Approval and Consent to Participate}

Not applicable.

\section{Consent for Publication}

Not applicable.

\section{Competing Interests}

The authors declare that they have no competing interests.

\section{Publisher's Note}

Springer Nature remains neutral with regard to jurisdictional claims in published maps and institutional affiliations.

\section{Author details \\ ${ }^{1}$ Department of Basic Sciences, Faculty of Veterinary Medicine, Ferdowsi University of Mashhad, Azadi Square, Mashhad, Iran. ${ }^{2}$ Division of Biotechnology, Faculty of Veterinary Medicine, Ferdowsi University of Mashhad, Azadi Square, Mashhad, Iran. ${ }^{3}$ Stem Cell Biology and Regenerative Medicine Research Group, Research Institute of Biotechnology, Ferdowsi University of Mashhad, Azadi Square, Mashhad, Iran.}

\section{Received: 21 August 2018 Accepted: 11 October 2018}

\section{Published online: 01 November 2018}

\section{References}

1. Carrieri C, Cimatti L, Biagioli M, Beugnet A, Zucchelli S, Fedele S, et al. Long non-coding antisense RNA controls Uchl1 translation through an embedded SINEB2 repeat. Nature. 2012;491:454-7.

2. Fatica A, Bozzoni I. Long non-coding RNAs: new players in cell differentiation and development. Nat Rev Genet. 2014;15:7-21.

3. Long $Y$, Wang $X$, Youmans DT, Cech TR. How do IncRNAs regulate transcription? Sci Adv. 2017;3:eaao2110.

4. Ghosal S, Das S, Chakrabarti J. Long noncoding RNAs: new players in the molecular mechanism for maintenance and differentiation of pluripotent stem cells. Stem Cells Dev. 2013;22:2240-53.

5. Goyal A, Myacheva K, Groß M, Klingenberg M, Duran Arqué B, Diederichs S. Challenges of CRISPR/Cas9 applications for long non-coding RNA genes. Nucleic Acids Res. 2017:45:e12.

6. Bassett AR, Akhtar A, Barlow DP, Bird AP, Brockdorff N, Duboule D, et al. Considerations when investigating IncRNA function in vivo. eLife. 2014;3: e03058

7. Boettcher M, McManus MT. Choosing the right tool for the job: RNAi, TALEN, or CRISPR. Mol Cell. 2015;58:575-85.
8. Nissan A, Stojadinovic A, Mitrani-Rosenbaum S, Halle D, Grinbaum R, Roistacher $\mathrm{M}$, et al. Colon cancer associated transcript-1: a novel RNA expressed in malignant and pre-malignant human tissues. Int J Cancer. 2012:130:1598-606.

9. Alaiyan B, llyayev N, Stojadinovic A, Izadjoo M, Roistacher M, Pavlov V, et al. Differential expression of colon cancer associated transcript1 (CCAT1) along the colonic adenoma-carcinoma sequence. BMC Cancer. 2013;13:196.

10. Yang F, Xue X, Bi J, Zheng L, Zhi K, Gu Y, et al. Long noncoding RNA CCAT1, which could be activated by c-Myc, promotes the progression of gastric carcinoma. J Cancer Res Clin Oncol. 2013;139:437-45.

11. Zhang Y, Ma M, Liu W, Ding W, Yu H. Enhanced expression of long noncoding RNA CARLo-5 is associated with the development of gastric cancer. Int J Clin Exp Pathol. 2014:7:8471-9.

12. Mizrahi I, Mazeh H, Grinbaum R, Beglaibter N, Wilschanski M, Pavlov V, et al. Colon Cancer associated Transcript-1 (CCAT1) expression in adenocarcinoma of the stomach. J Cancer. 2015;6:105-10.

13. Liu S-P, Yang J-X, Cao D-Y, Shen K. Identification of differentially expressed long non-coding RNAs in human ovarian cancer cells with different metastatic potentials. Cancer Biol Med. 2013:10:138-41.

14. Deng L, Yang S-B, Xu F-F, Zhang J-H. Long noncoding RNA CCAT1 promotes hepatocellular carcinoma progression by functioning as let-7 sponge. J Exp Clin Cancer Res. 2015;34:18.

15. Zhuang K, Wu Q, Jiang S, Yuan H, Huang S, Li H. CCAT1 promotes laryngeal squamous cell carcinoma cell proliferation and invasion. Am J Transl Res. 2016;8:4338-45

16. Cao Y, Shi H, Ren F, Jia Y, Zhang R. Long non-coding RNA CCAT1 promotes metastasis and poor prognosis in epithelial ovarian cancer. Exp Cell Res. 2017;359:185-94.

17. Gao R, Zhang R, Zhang C, Zhao L, Zhang Y. Long noncoding RNA CCAT1 promotes cell proliferation and metastasis in human medulloblastoma via MAPK pathway. Tumori. 2017;104:43-50.

18. Weidle UH, Birzele F, Kollmorgen G, Rüger R. Long non-coding RNAs and their role in metastasis. Cancer Genomics Proteomics. 2017;14:143-60.

19. Chen L, Wang W, Cao L, Li Z, Wang X. Long non-coding RNA CCAT1 acts as a competing endogenous RNA to regulate cell growth and differentiation in acute myeloid leukemia. Mol Cells. 2016:39:330-6.

20. Dou C, Sun L, Jin X, Han M, Zhang B, Li T. Long non-coding RNA colon cancer-associated transcript 1 functions as a competing endogenous RNA to regulate cyclin-dependent kinase 1 expression by sponging miR-490-3p in hepatocellular carcinoma progression. Tumour Biol J Int Soc Oncodevelopmental Biol Med. 2017:39:1010428317697572.

21. Younger ST, Rinn JL. 'Lnc'-ing enhancers to MYC regulation. Cell Res. 2014 24:643-4.

22. Wang Z-H, Guo X-Q, Zhang Q-S, Zhang J-L, Duan Y-L, Li G-F, et al. Long non-coding RNA CCAT1 promotes glioma cell proliferation via inhibiting microRNA-410. Biochem Biophys Res Commun. 2016;480:715-20.

23. Lennox KA, Behlke MA. Cellular localization of long non-coding RNAs affects silencing by RNAi more than by antisense oligonucleotides. Nucleic Acids Res. 2016:44:863-77.

24. Suzuki K, Tsunekawa Y, Hernandez-Benitez R, Wu J, Zhu J, Kim EJ, et al. In vivo genome editing via CRISPR/Cas9 mediated homology-independent targeted integration. Nature. 2016;540:144-9.

25. Xiang J-F, Yin Q-F, Chen T, Zhang Y, Zhang X-O, Wu Z, et al. Human colorectal cancer-specific CCAT1-L IncRNA regulates long-range chromatin interactions at the MYC locus. Cell Res. 2014;24:513.

26. Guadamillas MC, Cerezo A, Del Pozo MA. Overcoming anoikis--pathways to anchorage-independent growth in cancer. J Cell Sci. 2011;124:3189-97.

27. Quent VMC, Loessner D, Friis T, Reichert JC, Hutmacher DW. Discrepancies between metabolic activity and DNA content as tool to assess cell proliferation in cancer research. J Cell Mol Med. 2010;14:1003-13.

28. Chan GKY, Kleinheinz TL, Peterson D, Moffat JG. A simple high-content cell cycle assay reveals frequent discrepancies between cell number and ATP and MTS proliferation assays. PLoS One. 2013;8:e63583

29. Han J, Zhang J, Chen L, Shen B, Zhou J, Hu B, et al. Efficient in vivo deletion of a large imprinted IncRNA by CRISPR/Cas9. RNA Biol. 2014;11:829-35.

30. Paralkar VR, Taborda CC, Huang P, Yao Y, Kossenkov AV, Prasad R, et al. Unlinking an IncRNA from its associated cis element. Mol Cell. 2016;62: 104-10.

31. Yin Y, Yan P, Lu J, Song G, Zhu Y, Li Z, et al. Opposing roles for the IncRNA haunt and its genomic locus in regulating HOXA gene activation during embryonic stem cell differentiation. Cell Stem Cell. 2015;16:504-16. 
32. Zhang E, Han L, Yin D, He X, Hong L, Si X, et al. H3K27 acetylation activatedlong non-coding RNA CCAT1 affects cell proliferation and migration by regulating SPRY4 and HOXB13 expression in esophageal squamous cell carcinoma. Nucleic Acids Res. 2017:45:3086-101.

33. Mooi JK, Luk IY, Mariadason JM. Cell line models of molecular subtypes of colorectal Cancer. Methods Mol Biol Clifton NJ. 2018;1765:3-26.

34. Trapnell C, Hendrickson DG, Sauvageau M, Goff L, Rinn JL, Pachter L. Differential analysis of gene regulation at transcript resolution with RNA-seq. Nat Biotechnol. 2013;31:46-53.

35. Huang DW, Sherman BT, Lempicki RA. Systematic and integrative analysis of large gene lists using DAVID bioinformatics resources. Nat Protoc. 2009;4: 44-57.

36. Huang DW, Sherman BT, Lempicki RA. Bioinformatics enrichment tools: paths toward the comprehensive functional analysis of large gene lists. Nucleic Acids Res. 2009:37:1-13.

37. Horibata S, Vo TV, Subramanian V, Thompson PR, Coonrod SA. Utilization of the soft agar Colony formation assay to identify inhibitors of Tumorigenicity in breast Cancer cells. J Vis Exp. 2015;99:e52727.

38. Schneider CA, Rasband WS, Eliceiri KW. NIH image to ImageJ: 25 years of image analysis. Nat Methods. 2012;9:671-5.

Ready to submit your research? Choose BMC and benefit from:

- fast, convenient online submission

- thorough peer review by experienced researchers in your field

- rapid publication on acceptance

- support for research data, including large and complex data types

- gold Open Access which fosters wider collaboration and increased citations

- maximum visibility for your research: over $100 \mathrm{M}$ website views per year

At $\mathrm{BMC}$, research is always in progress.

Learn more biomedcentral.com/submissions 